\title{
Spectral Investigation of Laser Plasma Sources for X-Ray Coherence Tomography
}

\begin{abstract}
A. Arikkatt*, P. Wachulak, A. Bartnik and H. Fiedorowicz
Institute of Optoelectronics, Military University of Technology, S. Kaliskiego 2, 00-908 Warsaw, Poland

We present spectral investigations of laser-plasma sources, in the extreme ultraviolet (EUV) and soft X-ray (SXR) regions from high- $Z$ gases, dedicated for the X-ray coherence tomography experiments. The laser pulses of $4 \mathrm{~ns}$ and $650 \mathrm{~mJ}$ are used to produce plasma in a double gas puff target. The spectral investigation is done using 3 different spectrometers, spanning $1 \mathrm{~nm}$ to $70 \mathrm{~nm}$ of the EUV/SXR spectral region. Grazing incidence spectrometers are used in the ranges of 1-5 nm and 10-70 nm, and transmission grating spectrometer is used in the range of $4-16 \mathrm{~nm}$. Specific wavelength bands were identified which are suitable for SXR and EUV XCT measurements. The compact size of the experimental setup of about $\approx 1.5 \times 1.5 \mathrm{~m}^{2}$ makes it suitable for laboratory environments.
\end{abstract}

DOI: 10.12693/APhysPolA.137.48

PACS/topics: 32.30.Rj, 42.62.Fi, 52.38.-r, 52.50.Dg, 52.50.Jm, 87.64.Gb

\section{Introduction}

SXR/EUV sources are identified to have potential in high resolution imaging techniques due to their lower wavelengths. Broadband emission from laserplasma sources allow polychromatic imaging techniques to be implemented, such as X-ray coherence tomography (XCT) [1]. The axial resolution of the reconstructed 3D images in XCT are determined by the spectral characteristics of radiation sources. The axial resolution is directly related to the coherence length and thus inversely proportional to the spectral width of the emission. The broadband emission required for the XCT can be achieved using high- $Z$ gases as laser targets. The spectral emission from Xe and $\mathrm{Kr}$ has been studied for different applications $[2,3]$.

In the present paper, we explore the possibility to use spectral emissions from $\operatorname{Kr}(Z=36)$ and $\operatorname{Xe}(Z=54)$ gas targets and KrXe mixture. The broadband emission from high- $Z$ gases obtained through a laser-matter interaction is sufficiently broad for the XCT measurements. We detail the experimental setup used for the spectral measurements from 1-70 $\mathrm{nm}$. The results are discussed, and conclusions are made in the succeeding sections.

\section{Experimental setup}

The experimental setup for laser plasma SXR-EUV source is shown in Fig. 1. The setup consists of Nd:YAG pumping laser, source vacuum chamber with double gas jet valve, and three different spectrometers for different wavelength ranges in EUV/SXR region. The Nd:YAG laser (NL303HT, EKSPLA, Lithuania) with an operating wavelength of $1064 \mathrm{~nm}$ is used to produce a laser

*corresponding author; e-mail: antony.arikkatt@wat.edu.pl

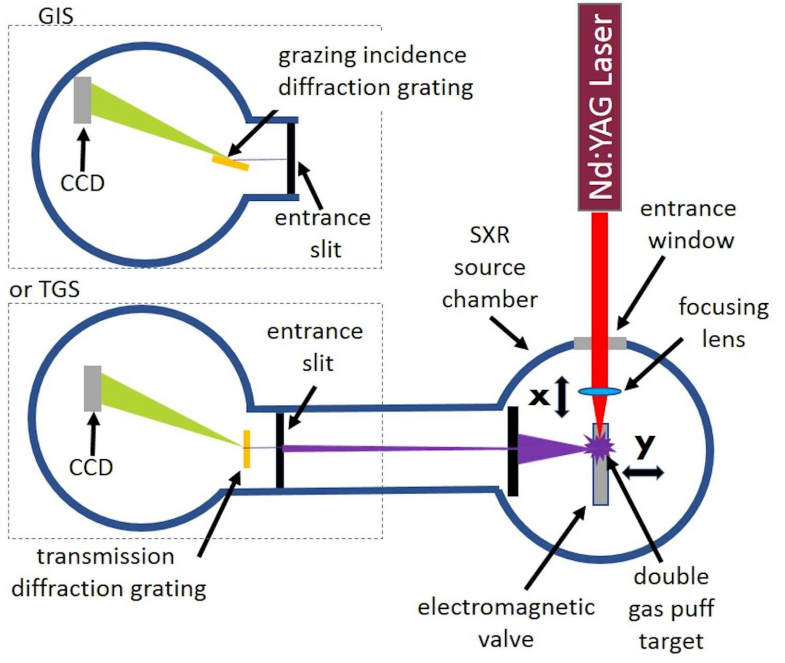

Fig. 1. Schematic of experimental setup with TGS and GIS.

pulse of duration $4 \mathrm{~ns}$ and energy $650 \mathrm{~mJ}$ with a repetition rate of $10 \mathrm{~Hz} .100$ laser pulses were used to acquire spectra.

The double stream gas target is produced by an electromagnetic valve, located inside the source chamber. The gases are injected into the laser-matter interaction region using a concentric double nozzle. The inner nozzle has a diameter of $0.4 \mathrm{~mm}$ and is supplied with a target gas to which a specific emission spectrum can be attributed. The outer, ring-shaped nozzle with $0.7 \mathrm{~mm}$ to $1.5 \mathrm{~mm}$ in diameter is supplied with helium gas to confine the target gas. The laser beam is focused at $1.5 \mathrm{~mm}$ above the gas nozzle using a lens with $27 \mathrm{~mm}$ focal length. Both nozzle and lens are placed on translational stages and optimized for maximum output. More details about the EUV/SXR LPP source and its optimization can be found in Ref. [4]. The target gas is supplied at 5 bar pressure and helium gas is supplied at 6 bar backing pressure. 
Three different spectrometers are used to measure emission spectra from the plasma in the range of $1-70 \mathrm{~nm}$. Schematic of grazing incidence spectrometer (GIS) and transmission grating spectrometer (TGS) is provided in Fig. 1. GIS is used to measure the spectra in the wavelength ranges 1-5 $\mathrm{nm}$ (SXR) and $10-70 \mathrm{~nm}$ (EUV II). The radiation in the wavelength range $4-16 \mathrm{~nm}$ (EUV I) is measured using a TGS. To measure SXR radiation in the range $1-5 \mathrm{~nm}$, an entrance slit of $12 \mu \mathrm{m}$ and a grazing incidence diffraction grating of 2400 lines $/ \mathrm{mm}$ are used. A back-illuminated CCD camera (greateyes GmbH, Germany) with $2 \mathrm{k} \times 2 \mathrm{k}$ resolution and pixel size of $13 \times 13 \mu \mathrm{m}^{2}$, cooled to $-20^{\circ} \mathrm{C}$, is used to record the spectra from high- $Z$ gases such as $\mathrm{Kr}$, Xe, and their mixture. In the EUV I range a $30 \mu \mathrm{m}$ entrance slit and a transmission diffraction grating of 5000 lines $/ \mathrm{mm}$ are used. For spectral data recording of this spectrometer, a back-illuminated CCD camera (Andor, UK) with $1 \mathrm{k} \times 1 \mathrm{k}$ resolution and a pixel size of $13 \times 13 \mu \mathrm{m}^{2}$, cooled down to $0^{\circ} \mathrm{C}$, is used. In EUV II spectrometer, an entrance slit of $25 \mu \mathrm{m}$ and grazing incidence diffraction grating of 450 lines $/ \mathrm{mm}$ is used. In this case, a backilluminated CCD camera (Andor, UK) with a resolution $1 \mathrm{k} \times 1 \mathrm{k}$ and a pixel size of $13 \times 13 \mu \mathrm{m}^{2}$, cooled down to $-20^{\circ} \mathrm{C}$ is used to record the spectral output.

\section{Results and discussion}

The spectral emissions in the range of 1 to $70 \mathrm{~nm}$ for $\mathrm{Kr}$, Xe gases and $\mathrm{KrXe}$ (v/v 90/10\%) mixture were measured. Such gases were used as possible candidate targets for laser-matter interactions dedicated to the XCT experiment. The XCT requires large bandwidth emission to obtain small coherence length $\left(l_{c}\right)[5]$ in the range of (preferably) a few nanometers [1] allowing for high axial resolution reconstruction of tomographic images. The spectral bands were identified according to national institute of standards and technology (NIST) data [6]. The spectral bands were identified with the ionization levels and spectral lines were identified with the transition wavelengths.

The spectral emissions in the EUV/SXR wavelength range from krypton gas target are provided in Fig. 2a-c. We could identify that krypton has broadband emissions spanning $\approx 0.35 \mathrm{~nm}$ centred at $4.5,4.9$, and $5.4 \mathrm{~nm}$ wavelengths superimposed on top of a quasi-continuum spanning from 2.5 to $7 \mathrm{~nm}$, resulting in $l_{c} \approx 1.8 \mathrm{~nm}$. These are identified as emissions related to the ionization levels of $\operatorname{Kr}(\mathrm{XI}), \operatorname{Kr}(\mathrm{XII})$, and $\operatorname{Kr}(\mathrm{XIII})$ [7]. Recently, a preliminary experiment related to SXR XCT measurements was performed using this emission [1]. A much wider band was identified starting from 8.8 to $11 \mathrm{~nm}$, which corresponds to ionization levels of $\operatorname{Kr}(\mathrm{X})$ to $\operatorname{Kr}(\mathrm{XIII})$. In the EUV II range we could identify a significant output from 10 to $12 \mathrm{~nm}$ which agrees with EUV I measurements. This emission could find XCT applications in the EUV range. Bands identified are corresponding to the transitions in $\mathrm{Kr}$ plasma from $\mathrm{Kr}(\mathrm{IX})$ to $\mathrm{Kr}$ (XVIII) ions.
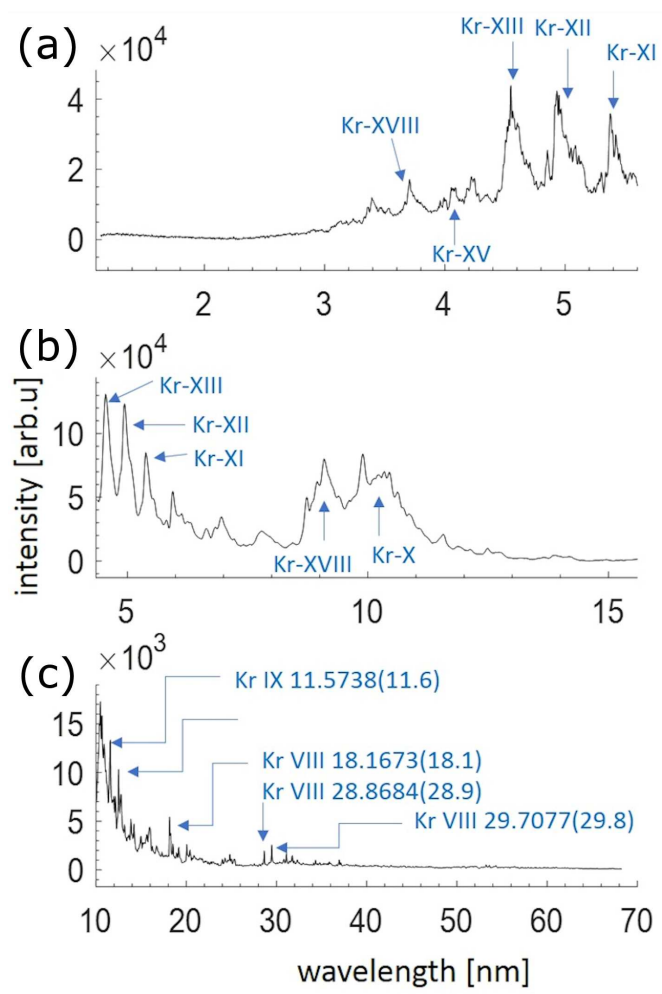

Fig. 2. Spectral investigation of EUV/SXR emission from krypton gas target in wavelength ranges $1-5 \mathrm{~nm}$ (a), 4-16 nm (b), 10-70 nm (c).
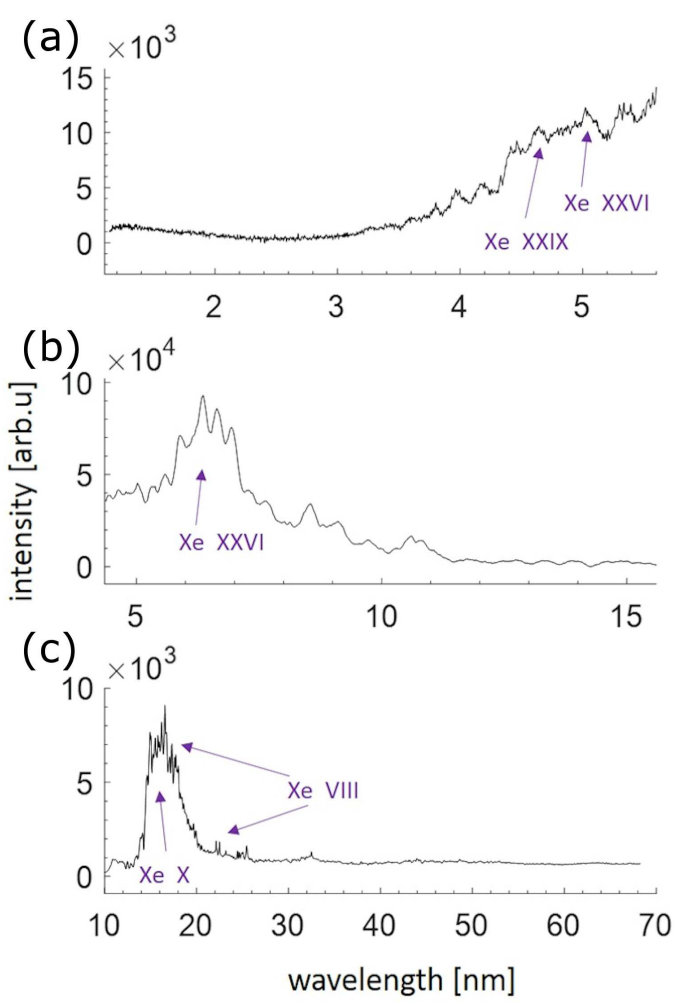

Fig. 3. Spectral investigation of EUV/SXR emission from xenon gas target in the wavelength ranges $1-5 \mathrm{~nm}$ (a), 4-16 nm (b), 10-70 nm (c). 

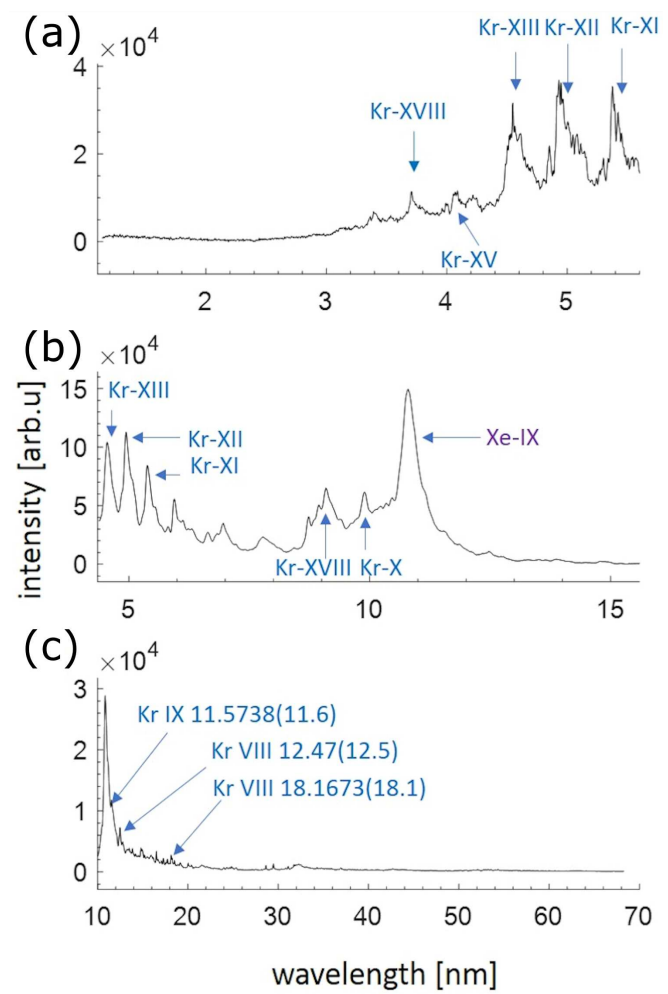

Fig. 4. Spectral investigation of EUV/SXR emission from krypton-xenon mixture gas target in wavelength ranges 1-5 $\mathrm{nm}$ (a), 4-16 $\mathrm{nm}$ (b), 10-70 $\mathrm{nm}$ (c).

Spectral emission from Xe $(Z=54)$ gas is presented in Fig. 3a-c. Owing to much higher atomic number, it is expected that the emission bandwidth in the EUV/SXR region from Xe plasma will be wider comparing to that from Kr plasma. Indeed, a significant broadband emission is detected in the range of 3.5 to $7.5 \mathrm{~nm}$ wavelength. According to the NIST database, it is identified that the predominant emissions from Xe plasma in that wavelength range can be attributed to the $\mathrm{Xe}(\mathrm{XXIX})$ and $\mathrm{Xe}(\mathrm{XXVI})$ ions.

Moreover, in the wavelength range from 14 to $20 \mathrm{~nm}$, $\mathrm{Xe}$ plasma has significant emissions in the range from $15 \mathrm{~nm}$ to $16.3 \mathrm{~nm}$, related to $\mathrm{Xe}(\mathrm{IX}), \mathrm{Xe}(\mathrm{X})$, and $\mathrm{Xe}(\mathrm{XI})$ ions, and in the range 16.6 to $18.5 \mathrm{~nm}$ related to the $\mathrm{Xe}(\mathrm{VIII})$ ions. Such emission band from 15 to $18.5 \mathrm{~nm}$, resulting in $l_{c}$ of $\approx 35 \mathrm{~nm}$, can also be interesting from the point of view of the EUV XCT.

Spectral emission characteristics of a mixture of $\mathrm{KrXe}$ is presented in Fig. $4 \mathrm{a}-\mathrm{c}$. Since this mixture is mostly $\mathrm{Kr}$, the emission spectrum of such mixture in the SXR range is dominated by the radiation from $\mathrm{Kr}$ plasma. Moreover, an additional high-intensity emission, related to $\mathrm{Xe}(\mathrm{IX})$ ions in the range from $10.6 \mathrm{~nm}$ to $11.4 \mathrm{~nm}$, is also identified due to the low rate of reabsorption of Xe, since its concentration is low in the mixture. This emission from Xe(IX) ions (10.6-11.4 nm) together with the emission from $\mathrm{Kr}$ (XVIII) around $9 \mathrm{~nm}$ wavelength spans $\approx 2 \mathrm{~nm}$ around a central wavelength at $10 \mathrm{~nm}$ results in $l_{c}$ of $\approx 20 \mathrm{~nm}$ and may be suitable for EUV XCT.

\section{Conclusion}

EUV/SXR spectral emissions from LPP source, based on double stream $\mathrm{Kr} / \mathrm{He}, \mathrm{Xe} / \mathrm{He}$, and $\mathrm{KrXe} / \mathrm{He}$ gas puff targets are measured using three different spectrometers. The spectra are analysed and most suitable spectral ranges for XCT application are found out. The effects of mixture on spectral data were analysed.

The spectral characterization of such source is very important in calculation of maximum possible resolution of depth information that can be obtained from XCT experiments. The magnitude of the radiations is also important in terms of the time required for $\mathrm{XCT}$ experiments.

\section{Acknowledgments}

The research was funded by the National Science Centre (NCN) (2016/23/G/ST2/04319, UMO2015/19/ B/ST3/00435), European Union's Horizon 2020 research and innovation program Laserlab-Europe IV (654148). We also thank the referees for their useful and constructive comments.

\section{References}

[1] P. Wachulak, A. Bartnik, H. Fiedorowicz, Sci. Rep. 8, 8494 (2018).

[2] H. Fiedorowicz, A. Bartnik, M. Szczurek, et al., Opt. Commun. 163, 103 (1999).

[3] A. Sasaki, K. Nishihara, F. Koike, T. Kagawa, T. Nishikawa, K. Fujima, T. Kawamura, H. Furukawa, IEEE J. Sel. Top. Quant. Elec. 10, 1307 (2004).

[4] P. Wachulak, M. Duda, T. Fok, A. Bartnik, Z. Wang, Q. Huang, A. Sarzyński, A. Jancarek, H. Fiedorowicz, Materials 11, 1303 (2018).

[5] S. Fuchs, A. Blinne, C. Rödel, et al., Appl. Phys. B 106, 789 (2012).

[6] NIST, Online database for spectral lines.

[7] R.L. Kelly, J. Phys. Chem. Ref. Data 16, 651 (1987). 